\title{
$\mu$-Term Hybrid Inflation and Split Supersymmetry
}

\author{
Qaisar SHAFI ${ }^{* \dagger}$ \\ University of Delaware \\ E-mail: shafi@bartol.udel.edu
}

We summarize the results of a recent paper on MSSM $\mu$-term hybrid inflation which, in its minimal format with gravity mediated supersymmetry breaking, leads to split supersymmetry. The $\mu$ term is larger than the gravitino mass $m_{3 / 2}$, and successful inflation followed by reheating requires $m_{3 / 2} \gtrsim 10^{7} \mathrm{GeV}$. In this case the gravitino decays before the LSP neutralino freezes out. Assuming universal scalar masses of the same order as $m_{3 / 2}$, this leads to split supersymmetry. The LSP wino with mass $\sim 2 \mathrm{TeV}$ is a plausible dark matter candidate, the gluino in principle is accesible at the LHC, and the MSSM parameter $\tan \beta \approx 1.7$ in order to yield the required Higgs boson mass. The tensor to scalar ratio $r \gtrsim 0.001$.

18th International Conference From the Planck Scale to the Electroweak Scale 25-29 May 2015

Ioannina, Greece

\footnotetext{
* Speaker.

${ }^{\dagger}$ Based on work done in collaboration with Nobuchika Okada.
} 
Consider the following renormalizable superpotential, which is unique under a $U(1)$ R-symmetry [2]

$$
W=S\left(\kappa \bar{\Phi} \Phi-\kappa M^{2}+\lambda H_{u} H_{d}\right)
$$

Here $\Phi, \bar{\Phi}$ denote a conjugate pair of chiral superfields whose VEVs induce the spontaneous breaking of a gauge symmetry $G$, with supersymmetry unbroken. The dimensionless coefficients $\lambda$ and $\kappa$ can be made real by suitable field redefinitions. The superpotential $W$ and the $G$-singlet superfield $S$ are assigned unit R-charges, while the remaining superfields have zero R-charges.

In the case of global supersymmetry, the VEV of the scalar component of the $G$-singlet superfield $S$ is zero. However, taking into account supersymmetry breaking in supergravity, the $S$ field acquires a non-zero VEV proportional to the gravitino mass $m_{3 / 2}$. Following [1], we have included the term $\lambda S_{u} H_{d}$, which then induces the desired MSSM $\mu$ term. It was shown in [1] that to implement successful inflation, followed by the desired breaking of $G$ and the MSSM gauge symmetry unbroken, the parameter $\lambda>\kappa$. This additional term in the superpotential plays an essential role in the analysis.

With a minimal canonical Kähler potential, and taking into account radiative corrections and the linear soft supersymmetry breaking term, the inflationary potential is approximated by

$$
V(\phi)=m^{4}\left(1+\alpha \ln \left[\frac{\phi}{\phi_{0}}\right]\right)-2 \sqrt{2} m_{3 / 2} m^{2} \phi .
$$

Here the inflaton field $\phi$ is the real part of $S$, the renormalization scale $(Q)$ is set equal to the initial inflaton VEV $\phi_{0}$, and the imaginary part of $S$ is assumed to stay constant during inflation. The negative sign of the linear term is essential to generate the correct value for the spectral index. Note that in the absence of this linear term the scalar spectral index $n_{s}$ is predicted to lie close to 0.98 . Finally, $m=\sqrt{\kappa} M$, and the coefficient $\alpha \ll 1$ is given by

$$
\alpha=\frac{1}{4 \pi^{2}}\left(\lambda^{2}+\frac{N_{\Phi}}{2} \kappa^{2}\right)
$$

The coefficient $N_{\Phi}$ depends on the model under discussion. For instance, $N_{\Phi}=1$ if $G=\mathrm{U}(1)_{B-L}$, while it is 2 for $G=\mathrm{SU}(2)_{L} \times \mathrm{SU}(2)_{R} \times \mathrm{U}(1)_{B-L}$. Note that for inflaton field values close to $M$, the leading supergravity corrections are of order $\left(M / m_{P}\right)^{4}$ and therefore well suppressed, and also the quadratic soft supersymmetry breaking term $m_{\phi}^{2} \phi^{2}$ associated with the inflaton can be ignored relative to the liner term in Eq. (2) [2].

Where appropriate, we set

$$
V \simeq m^{4}, \quad V^{\prime}=m^{4} \frac{\alpha}{\phi}\left(1-\beta \frac{\phi}{\phi_{0}}\right), \quad V^{\prime \prime}=-m^{4} \frac{\alpha}{\phi^{2}},
$$

where

$$
\beta=\frac{2 \sqrt{2} m_{3 / 2} \phi_{0}}{\alpha m^{2}}
$$

is a dimensionless parameter of order unity. Note that $\beta=0$ if the linear soft supersymmetry breaking term is ignored. At the potential minimum, the inflaton VEV is given by $\langle\phi\rangle=m_{3 / 2} / \kappa$, 
which yields the MSSM $\mu$-term:

$$
\mu=\gamma m_{3 / 2}
$$

where $\gamma \equiv \lambda / \kappa>1$.

The well-known slow roll parameters are given by

$$
\varepsilon=\frac{1}{2}\left(\frac{V^{\prime}}{V}\right)^{2} \simeq \frac{1}{2} \frac{\alpha^{2}}{\phi^{2}}\left(1-\beta \frac{\phi}{\phi_{0}}\right)^{2}, \quad \eta=\frac{V^{\prime \prime}}{V} \simeq-\frac{\alpha}{\phi^{2}} .
$$

For convenience we have used units where the reduced Planck mass $m_{P}=2.43 \times 10^{18} \mathrm{GeV}$ is set equal to unity. For $\alpha \ll 1,|\eta| \gg \varepsilon$. The end of inflation is determined by $|\eta|=1$, so that the inflaton value at the end of inflation is given by $\phi_{e} \simeq \sqrt{\alpha}$.

The number of e-foldings is estimated to be

$$
N=\int_{\phi_{e}}^{\phi_{0}} \frac{V}{V^{\prime}} d \phi \simeq \frac{\phi_{0}^{2}}{\alpha} \int_{\phi_{e} / \phi_{0}}^{1} \frac{x d x}{1-\beta x} \simeq \frac{\phi_{0}^{2}}{\alpha}\left(-\frac{1}{\beta}-\frac{\ln (1-\beta)}{\beta^{2}}\right) \equiv \frac{\phi_{0}^{2}}{\alpha} f(\beta),
$$

where we have used the approximation $\phi_{e} / \phi_{0}=0$ in the integral. For the scalar spectral index, we obtain

$$
n_{s}=1-6 \varepsilon\left(\phi_{0}\right)+2 \eta\left(\phi_{0}\right) \simeq 1+2 \eta\left(\phi_{0}\right) \simeq 1-\frac{2}{N} f(\beta)
$$

In particular, for $N=60$ and $\beta=0, f(\beta)=1 / 2$ and we obtain the well known result of Ref. [2] that $n_{s}=1-1 / N \simeq 0.983$. To obtain the spectral index in the range of $0.955 \leq n_{s} \leq 0.977$ which is indicated by $1 \sigma$ region in the Planck 2015 results, the parameter $\beta$ lies in the range $0.83 \geq \beta \geq 0.41$, with $f(\beta)$ close to unity. A plot of $n_{s}$ versus $\beta$ is shown in Fig. 1 , along with $1 \sigma$ (dotted lines) and $2 \sigma$ (dashed lines) limits by the Planck 2015 results [2].

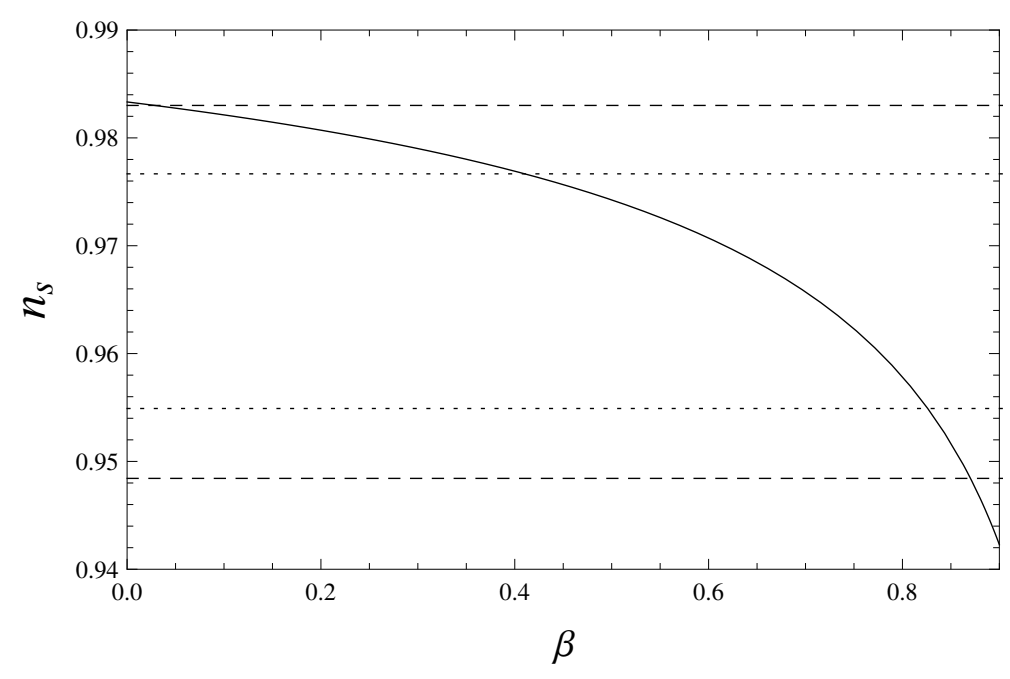

Figure 1: Spectral index $n_{s}$ vs. $\beta$ as defined in Eq. (5). The region between the two dotted (dashed) lines corresponds to $1 \sigma(2 \sigma)$ limit obtained by Planck 2015 [2]. 
The tensor-to-scalar ratio is given by

$$
r=16 \varepsilon\left(\phi_{0}\right) \simeq 4 \alpha(1-\beta)^{2}\left(1-n_{s}\right)
$$

where we have used Eqs. (7) and (9). With $0.83 \geq \beta \geq 0.41$ which yields the $n_{s}$ prediction within $1 \sigma$ limit of the Planck 2015 results, we find

$$
r=(0.00014-0.00082) \times\left(\lambda^{2}+\frac{N_{\Phi}}{2} \kappa^{2}\right) .
$$

To proceed further we employ the power spectrum whose magnitude has been estimated (Planck 2015) to be

$$
\Delta_{\mathscr{R}}^{2}=\frac{1}{24 \pi^{2}} \frac{V\left(\phi_{0}\right)}{\varepsilon\left(\phi_{0}\right)}=2.20 \times 10^{-9} .
$$

Utilizing $V \simeq m^{4}$, and Eqs. (7) and (8), we find the following expression for the gauge symmetry breaking scale $\mathrm{M}$ :

$$
M \simeq 0.0032 \times \sqrt{\tilde{\gamma}}\left(\frac{60}{N}\right)^{1 / 4}(1-\beta)^{1 / 2} f(\beta)^{1 / 4},
$$

where $\tilde{\gamma}=\sqrt{\gamma^{2}+N_{\Phi} / 2}$. Setting $N_{0}=60$ and $0.83 \geq \beta \geq 0.41$, we estimate the symmetry breaking scale $M$ :

$$
M[\mathrm{GeV}]=(3.5-5.5) \times 10^{15} \sqrt{\tilde{\gamma}}
$$

The ratio $\phi_{0} / M$ is given by

$$
\phi_{0} / M \simeq 380 \times \kappa \sqrt{\tilde{\gamma}}\left(\frac{N}{60}\right)^{3 / 4}(1-\beta)^{-1 / 2} f(\beta)^{-3 / 4} \simeq(650-730) \times \kappa \sqrt{\tilde{\gamma}} .
$$

For $0.83 \geq \beta \geq 0.41$, and requiring this ratio to be greater than unity,

$$
\kappa \sqrt{\tilde{\gamma}}>(1.4-1.5) \times 10^{-3} .
$$

The inflaton decay width is estimated to be

$$
\Gamma\left(\phi_{0} \rightarrow \tilde{H}_{u} \tilde{H}_{d}\right)=\frac{\lambda^{2}}{8 \pi} m_{\phi},
$$

where $m_{\phi}=\sqrt{2} \kappa M$ is the inflaton mass. Using $\Gamma=H=T_{R H}^{2} \sqrt{\frac{\pi^{2}}{90} g_{\star}}$ with $g_{\star}=228.75$ for the MSSM, we find

$$
T_{R H}[\mathrm{GeV}] \simeq 1.5 \times 10^{16}(\kappa \sqrt{\tilde{\gamma}})^{3 / 2} \frac{\gamma}{\sqrt{\tilde{\gamma}}}\left(\frac{228.75}{g_{\star}}\right)\left(\frac{60}{N_{0}}\right)^{1 / 8}(1-\beta)^{1 / 4} f(\beta)^{1 / 8}
$$

The condition, $\phi_{0} / M>1$, leads to

$$
T_{R H}[\mathrm{GeV}]>(5.0-7.5) \times 10^{11} \frac{\gamma}{\sqrt{\gamma}}
$$


for the $1 \sigma$ limit from Planck 2015. Using $\tilde{\gamma}=\sqrt{\gamma^{2}+N_{\Phi} / 2}$ and $\gamma>1$, we obtain the lower bound $T_{R H} \gtrsim(5.0-7.5) \times 10^{11} \mathrm{GeV}$.

To avoid the constraint on the neutralino abundance from gravitino decay, assume that the LSP neutralino is still in thermal equilibrium when the gravitino decays. In this case, the LSP neutralino abundance is not related to the gravitino yield. Using a typical value of the ratio $x_{F} \equiv m_{\tilde{\chi}^{0}} / T_{F} \simeq 20$, where $T_{F}$ is the freeze out temperature of the LSP neutralino, this occurs for the gravitino lifetime,

$$
\tau_{G} \lesssim 4 \times 10^{-10}\left(\frac{1 \mathrm{TeV}}{m_{\tilde{\chi}^{0}}}\right)^{2},
$$

which yields

$$
m_{3 / 2} \gtrsim 4.6 \times 10^{7} \mathrm{GeV}\left(\frac{m_{\tilde{\chi}^{0}}}{2 \mathrm{TeV}}\right)^{2 / 3}
$$

Therefore, our cosmological scenario favors a gravitino mass at an intermediate scale.

In our $\mu$-term generation mechanism, $\mu=\gamma m_{3 / 2}$ with $\gamma \gtrsim 1$. To implement the electroweak symmetry breaking, the soft supersymmetry breaking mass for the MSSM Higgs doublets should satisfy $\left|m_{0}^{2}\right| \gtrsim \mu^{2}$. Thus, the soft scalar mass parameter $m_{0}$ also lies at an intermediate scale. On the other hand, the mass scale of the dark matter neutralino is of order $100 \mathrm{GeV}-\mathrm{TeV}$ to reproduce the observed relic abundance. Therefore, the split SUSY scenario [2] is compatible with our scheme, and we expect a hierarchy between the soft supersymmetry breaking scalar masses and the gaugino masses [A special structure in supergravity is crucial to realize this mass splitting [2]]. In split SUSY with a large $\mu$, an LSP wino with mass around $2 \mathrm{TeV}$ is the simplest dark matter candidate [2].

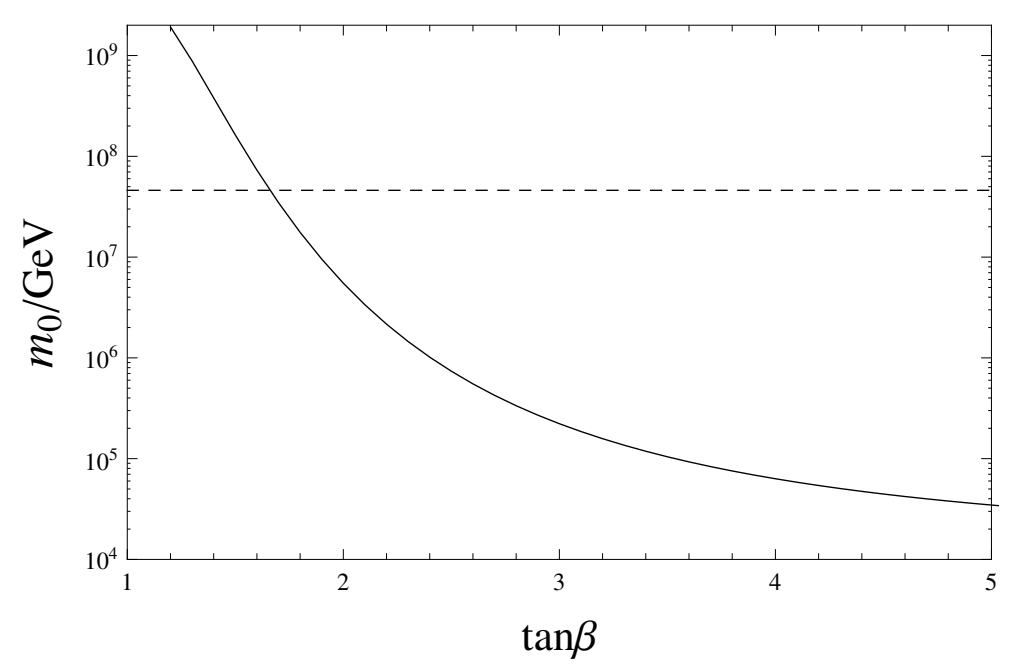

Figure 2: Soft scalar mass $\left(m_{0}\right)$ as a function of $\tan \beta$. Along the solid line, the Higgs boson mass $m_{h}=125.09 \mathrm{GeV}$ is realized for the top quark mass input $M_{t}=173.34 \mathrm{GeV}$. The horizontal dashed line denotes $m_{0}=4.6 \times 10^{7} \mathrm{GeV}$, corresponding to the lower bound on gravitino mass in Eq. (21) with $m_{\tilde{\chi}^{0}}=2 \mathrm{TeV}$. 
Let us fix the scalar mass scale in split SUSY so as to realize the observed Higgs boson mass. With the decoupling of heavy scalar fields at a typical mass scale $m_{0}$, split SUSY provides a boundary condition for the SM Higgs quartic coupling at $Q=m_{0}$ [2],

$$
\lambda_{h}\left(m_{0}\right)=\frac{1}{4}\left(g\left(m_{0}\right)^{2}+g^{\prime}\left(m_{0}\right)^{2}\right) \cos ^{2} 2 \beta .
$$

Our result is depicted in Fig. 2, where the Higgs boson mass $m_{h}=125.09 \mathrm{GeV}$ is reproduced along the solid line. For $m_{0}=4.6 \times 10^{7} \mathrm{GeV}$, corresponding to the lower bound on gravitino mass in Eq. (21) with $m_{\tilde{\chi}^{0}}=2 \mathrm{TeV}, m_{h}=125.09 \mathrm{GeV}$ is reproduced for $\tan \beta \simeq 1.7$.

Since the gauge group associated with inflation is an extension of the SM one, it may be natural to set the symmetry breaking scale $M$ equal to the gauge coupling unification scale, $M \simeq 2 \times 10^{16}$ $\mathrm{GeV}$, which is achieved with $\tilde{\gamma}=13-32$ in Eq. (14). In this case, we may choose $\lambda \simeq 1$ and $\kappa \simeq 0.1$, for which $\phi_{0} / M \gg 1$. For $\lambda=1$, the tensor-to-scalar ratio $r=(1.4-8.2) \times 10^{-4}$ for $0.83 \geq \beta \geq 0.41$ (see Eq. (11)). With somewhat larger values of $\lambda, r$ can be as high as $10^{-3}$.

In summary, the coupling of the MSSM Higgs doublets to the inflaton field induces the $\mu$-term and also leads to important predictions concerning the gravitino, neutralino dark matter, Higgs boson mass and low energy phenomenology. A consistent inflationary scenario requires a fairly large gravitino mass $\left(m_{3 / 2} \gtrsim 5 \times 10^{7} \mathrm{GeV}\right)$, and in the simplest scheme a $2 \mathrm{TeV}$ wino is a compelling thermal dark matter candidate. The soft supersymmetry breaking scalar masses are of order $10^{7}$ $\mathrm{GeV}$, with $\tan \beta \simeq 1.7$, in order to generate the desired $125 \mathrm{GeV}$ mass for the SM-like Higgs boson.

\section{Acknowledgments}

Q.S. thanks George Leontaris for his generous hospitality during the workshop.

\section{References}

[1] G. R. Dvali, G. Lazarides and Q. Shafi, Phys. Lett. B 424, 259 (1998) [hep-ph/9710314];

[2] N.Okada and Q. Shafi, [hep-ph/1506.01410v1] and all references therein. 